Supporting Information:

\title{
Depletion of atmospheric gaseous elemental mercury by plant uptake at Mt. Changbai, Northeast China
}

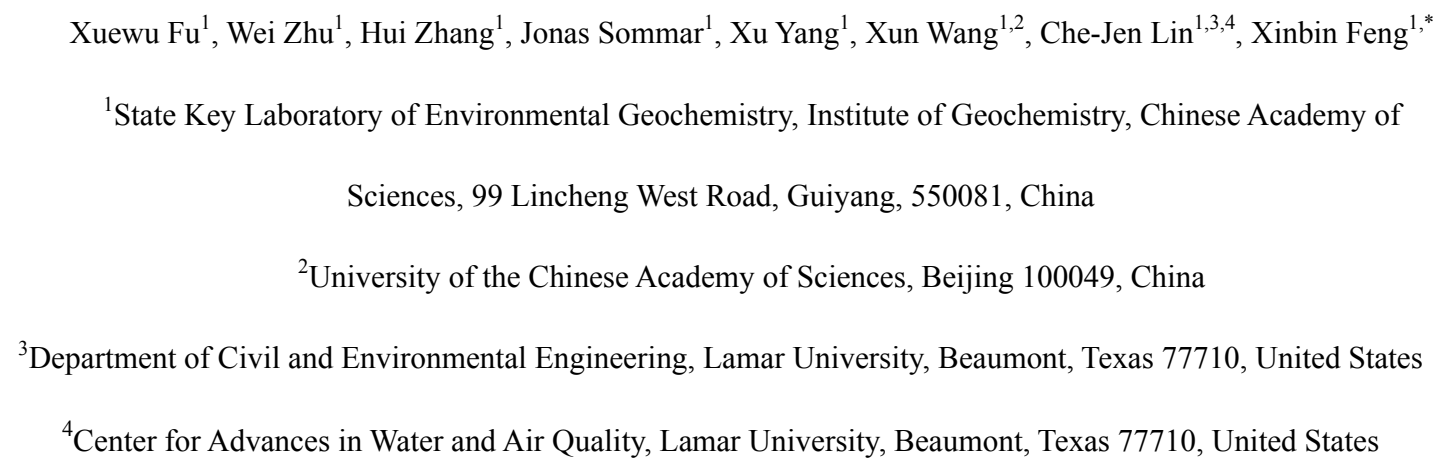

1 Supplementary Text. Page S1

1.1 Nocturnal boundary layer.

1.2 Simulations of atmospheric GEM at Mt. Changbai forest using a box model. .Page S1

1.3 The turbulent diffusivity $(\mathrm{Kc})$

Figure S1

.Page S3

Figure S2.

Figure S3 Page S5

Figure S4. .Page S6

Figure S5 .Page S7

Figure S6. .Page S8

Figure S7 .Page S9

Table S1 Page S10

Table S2 Page S11

Table S3 .Page S12

Table S4

Table S5

.Page S14

Reference. ..Page S15-S17 


\section{Supplementary Text}

\subsection{Nocturnal boundary layer}

The Nocturnal boundary layer (NBL) was calculated by Weather Research and Forecasting Model (WRF) 3.5 with two-way nested runs. The spatial resolution for course domain is $30 \mathrm{~km}$ with $100 \times 100$ grid cells, and for the nested domain is $10 \mathrm{~km}$ with $30 \times 30$ grid cells. As our studied site is $100-200 \mathrm{~km}$ away from Sea of Japan, we chose the MYJ scheme for NBL as earlier studies suggested that MYJ scheme was as first choice for marine atmospheric boundary layer simulations without $a$ priori information of atmospheric stability in the region of interest (Huang et al., 2013;Krogsaeter and Reuder, 2015). For other parameterizations, we selected Kain and Fritsch cumulus scheme for cumulus parameterization, Lin (Purdue) scheme for microphysics options RRTM scheme for Radiation Physics Options.

\subsection{Simulations of atmospheric GEM at Mt. Changbai forest using a box model}

A box model was applied to estimate the GEM concentration at the height of 24 $\mathrm{m}$ agl. Based on the measured characteristics of the GEM depletion events, the model assumes that vegetative uptake (in terms of dry deposition flux) is the only pathway for the GEM removal and chemical transformation is not included in the box modeling. A sensitivity analysis was performed on three parameters: (1) dry deposition flux (0-10 ng m $\mathrm{m}^{-2} \mathrm{~h}^{-1}$, the range of measured deposition flux using flux bags), (2) turbulent diffusivity of the atmosphere $\left(0.1-10 \mathrm{~cm} \mathrm{~s}^{-1}\right.$, typical value under low wind condition), and (3) a typical nocturnal boundary layer height (100 m agl). GEM concentration above the stable nocturnal boundary layer was assigned to 1.56 $\mathrm{ng} \mathrm{\textrm {m } ^ { - 3 }}$, the mean observed at $45 \mathrm{~m}$ during daytime when the vertical mixing is strong. The flux of vegetative uptake $\left(F_{C}\right)$ and the resulted concentration gradient was calculated based on the on the algorithm:

$$
F_{C}^{A G M}=-\underbrace{\frac{\kappa \cdot u_{*}}{\Phi_{H}\left(\varsigma_{1}\right)}}_{K_{C}} \cdot \frac{\partial C}{\partial \ln (z)}
$$

where $k$ is von Kármán constant $(\sim 0.41), u_{*}$ is the friction velocity $\left(\mathrm{m} \mathrm{s}^{-1}\right), \psi_{H}\left(\varsigma_{1}\right)$ is the integrated universal function for sensible heat to correct for deviations from the ideal logarithmic profile, $K_{C}$ term is the turbulent diffusivity $\left(\mathrm{m} \mathrm{s}^{-1}\right), C$ is the 
concentration parameter for GEM concentration $\left(\mathrm{ng} \mathrm{m}^{-3}\right), \mathrm{z}$ is the height parameter (m).

\subsection{The turbulent diffusivity $\left(K_{c}\right)$}

The flux-gradient approach (Kaimal and Finnigan, 1994) expresses a scalar flux ( $F$, e.g. ng $\left.\mathrm{m}^{-2} \mathrm{~s}^{-1}\right)$ as the product between the turbulent diffusivity $\left(K_{C}, \mathrm{~m}^{2} \mathrm{~s}^{-1}\right)$ and a concentration gradient $\left(\partial c / \partial z, \mathrm{ng} \mathrm{m}^{-4}\right)$ assuming that measurements are made within a vertical layer of constant flux that forms over homogeneous terrain:

$$
F=-K_{C} \cdot \frac{\partial c}{\partial z}=-\frac{u_{*} \cdot \kappa \cdot(z-d)}{\phi_{H}(\varsigma)} \cdot \frac{\partial c}{\partial z}=-\frac{u_{*} \cdot \kappa}{\phi_{H}(\varsigma)} \cdot \frac{\partial c}{\partial \ln z}
$$

where, $u_{*}$ is the friction velocity $\left(\mathrm{m} \mathrm{s}^{-1}\right), \kappa$ is the von Kármán constant (taken as 0.4), $\phi_{H}(\varsigma)$ is the diabatic influence function for heat (parameterized as a function of $\varsigma=(z-d) / L$, where $L$ is the Obukhov length), whereas $z$ and $d$ are the measurement and (canopy) displacement height (m) respectively. An empirical form of $\phi_{H}(\varsigma)$ is $0.95 / \sqrt{(1-11.6 \varsigma)}$ and $0.95+7.8 \varsigma$ for unstable $(\varsigma<0)$ and stable $(2>\varsigma \geq 0)$ atmospheric conditions respectively (Foken, 2008).

For flux-gradient measurements made within the roughness sublayer above the canopy height (i.e. $h_{\text {canopy }}<z<z_{*}$ ), Eq. 1 is not valid (underestimates the magnitude of scalar flux) and requires further correction following e.g. Garratt (Garratt, 1992) and Simpson et al. (Simpson et al., 1998):

$$
F=-\frac{u_{*} \cdot \kappa \cdot(z-d)}{\phi_{h}(\varsigma) \cdot \phi_{*}\left(z / z_{*}\right)} \cdot \frac{\partial c}{\partial z}
$$

In contrast to $\phi_{H}(\varsigma)$, the additional correction function $\phi_{*}\left(z / z_{*}\right)$ in Eq. 2 is independent of stability. A common type of parameterization for $\phi_{*}$ is $\exp \left[-0.7\left(1-\frac{z}{z_{*}}\right)\right]$ (Garratt, 1992). In-turn, the upper limit of the roughness sublayer $\left(z_{*}\right)$ can be estimated by $2 \cdot h_{\text {canopy }}-d$ 5 (Raupach, 1994). 
87 Figure S1. Map showing the location of Mt. Changbai forest and coal fired power plants in 88 Northeast Asia.

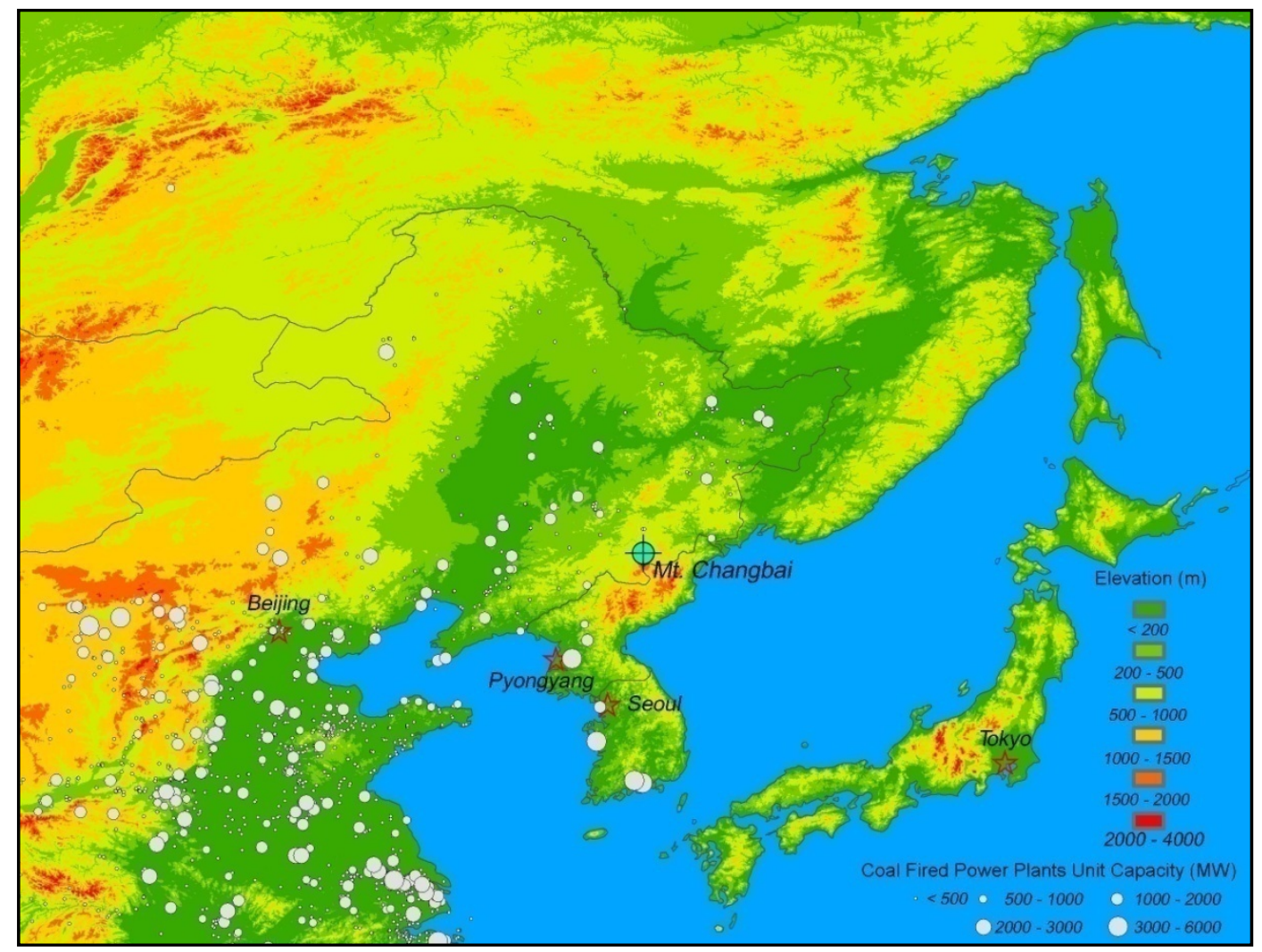

90

91

92 
Figure S2. GEM at $4 \mathrm{~m}$ agl in the clear spot measured by the Tekran speciation unit, $24 \mathrm{~m}$ agl $(\sim 3$

94

95

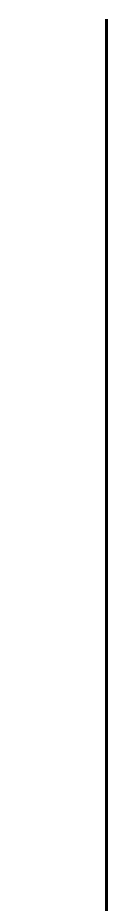

96

97

98

99

$\mathrm{m}$ above forest canopy, long-term GEM sampling site) and $45 \mathrm{~m}$ agl ( $\sim 24 \mathrm{~m}$ above forest canopy) measured by the Tekran 2537 from 10 to 14 July 2013.

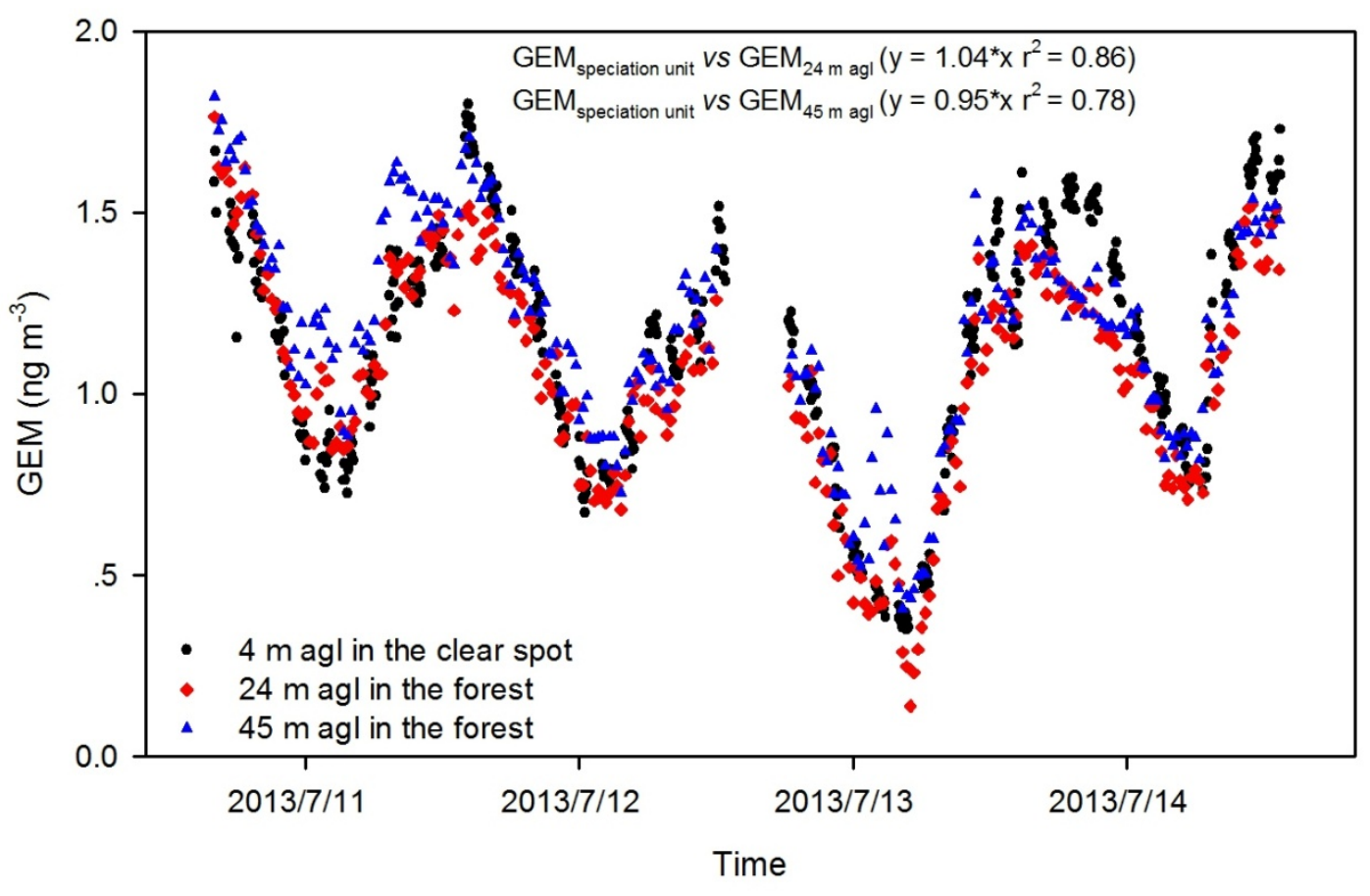


100 Figure S3. Time series of soil/air GEM flux and meteorological parameters in Mt. Changbai forest 101 in July 2013.

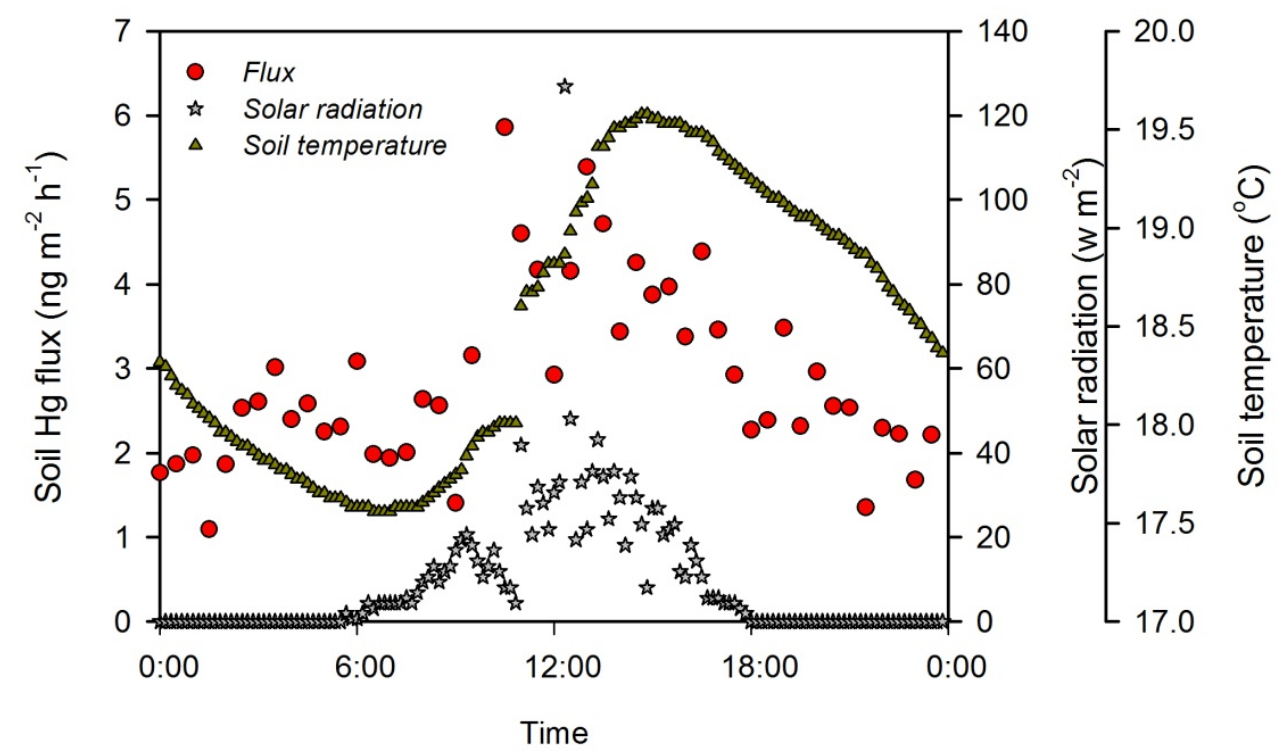

102

103

104

105 
106 Figure S4. Diurnal trends in vertical gradient of GEM concentrations between the height of 45-24

107 m, 24-10 m and 10-1m in Mt. Changbai forest from 10 to 15 July 2013.

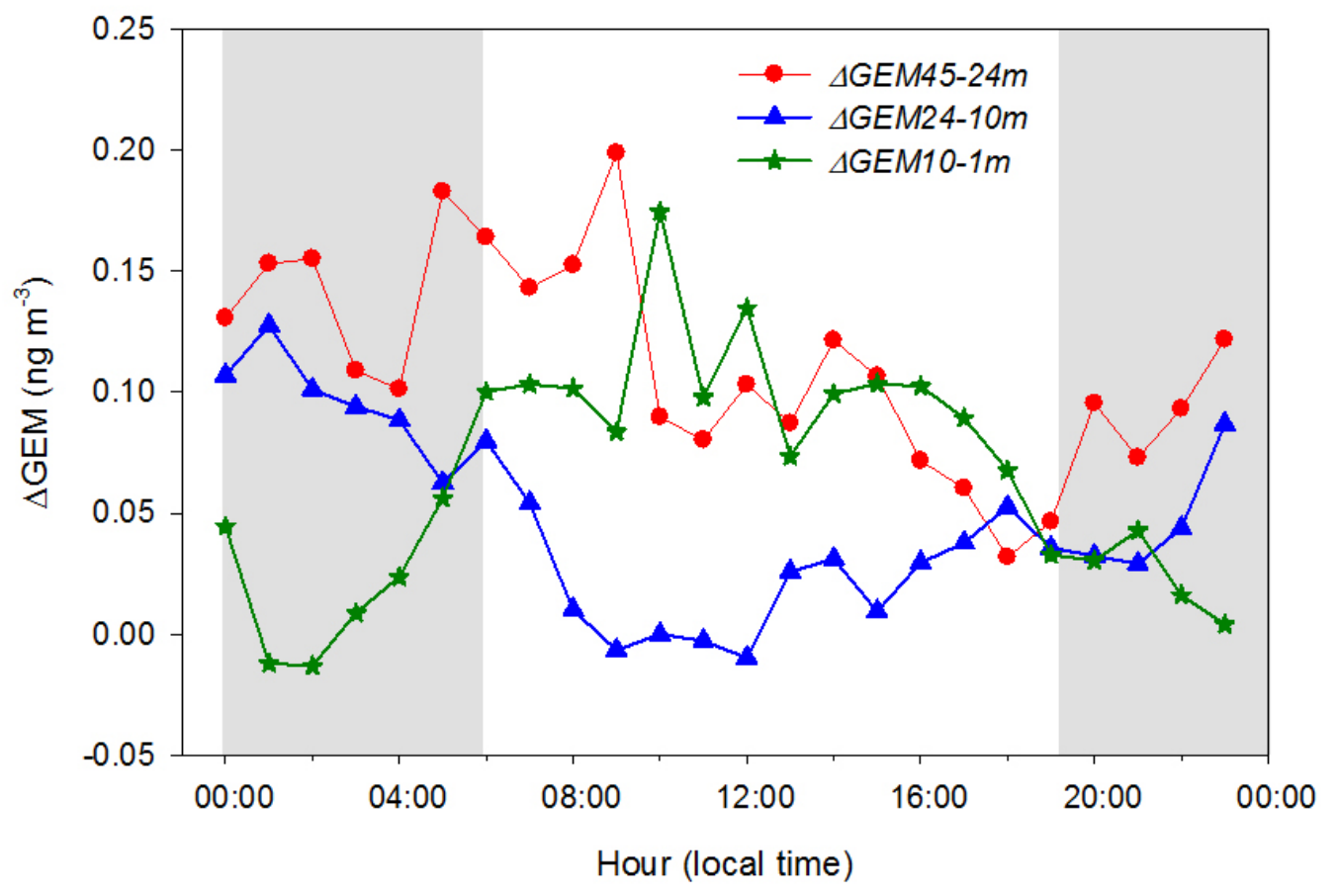

108 
112 Figure S5. Diurnal variations of air temperature (A), wind speed (B), relative humidity (RH, C) 113 and ozone concentrations (D) in Mt. Changbai forest in leaf-growing season from October 2008 to 114 December 2014.
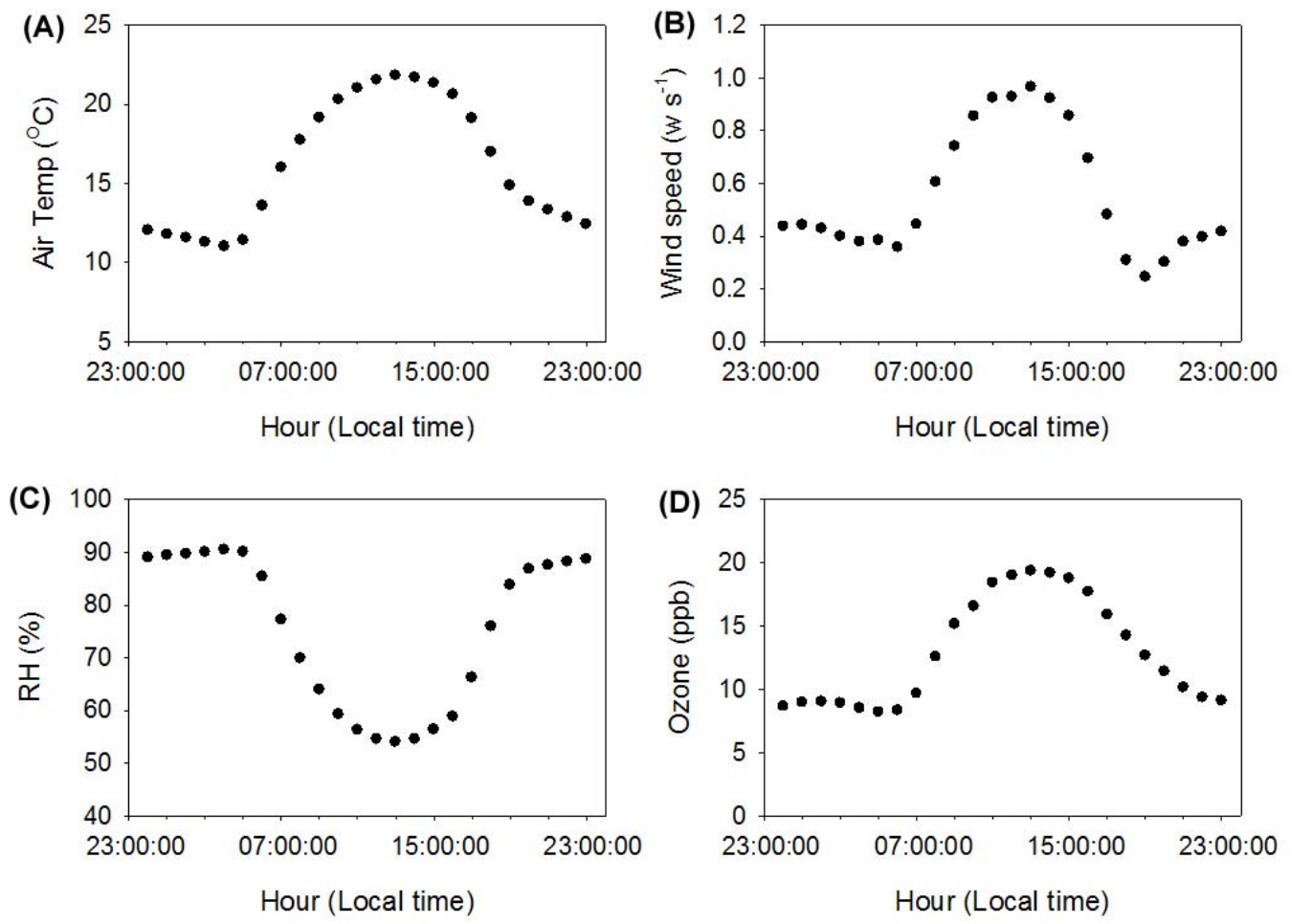
119 Figure S6. Temporal variation of the turbulent diffusivity at Mt. Changbai forest from 10 to 19 $120 \quad$ July 2013

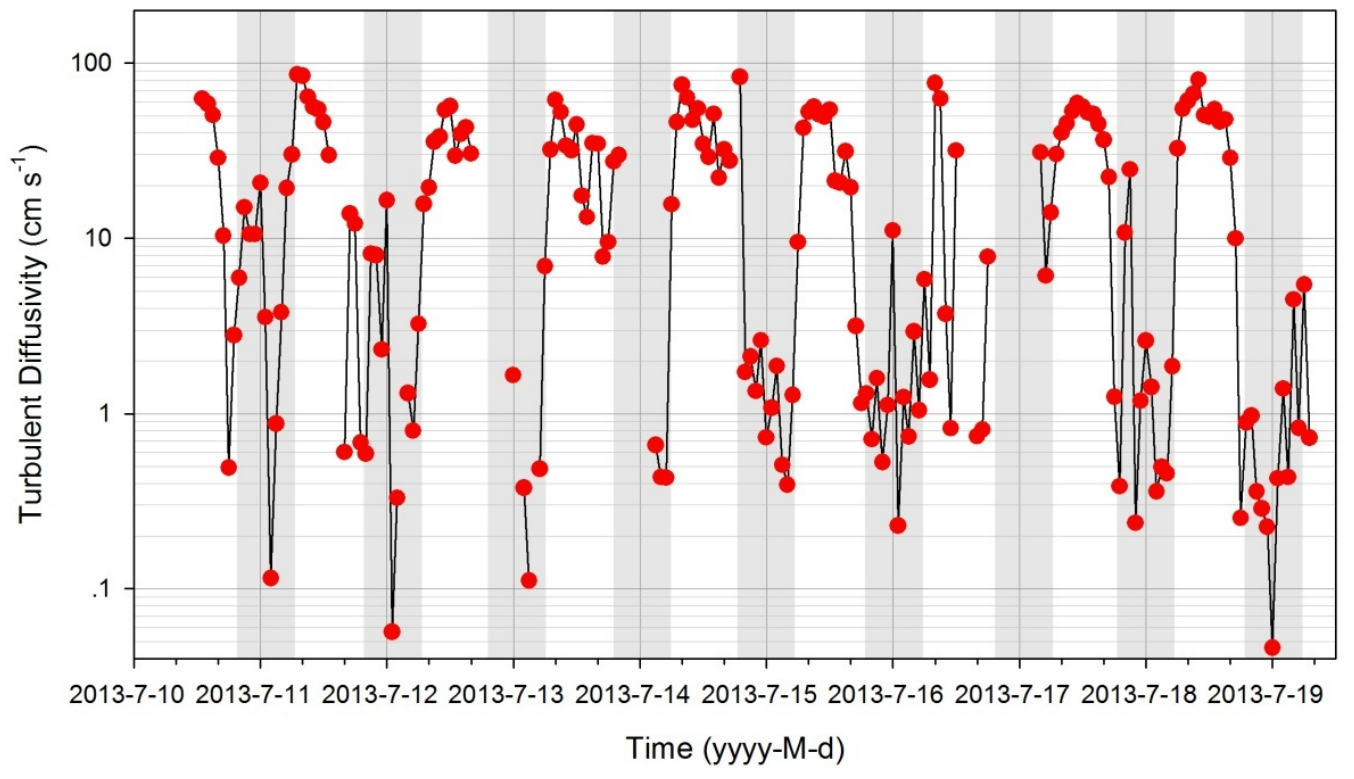

122 
125 Figure S7. Wind frequency distributions in leaf-growing season and non-leaf-growing season from 126 Aug 2009 to Jul 2013 (A), monthly mean wind speed from Aug 2009 to Jul 2013 (B), and Leaf 127 areas index in leaf-growing season during 2003-2005(C) (Shi et al., 2008).
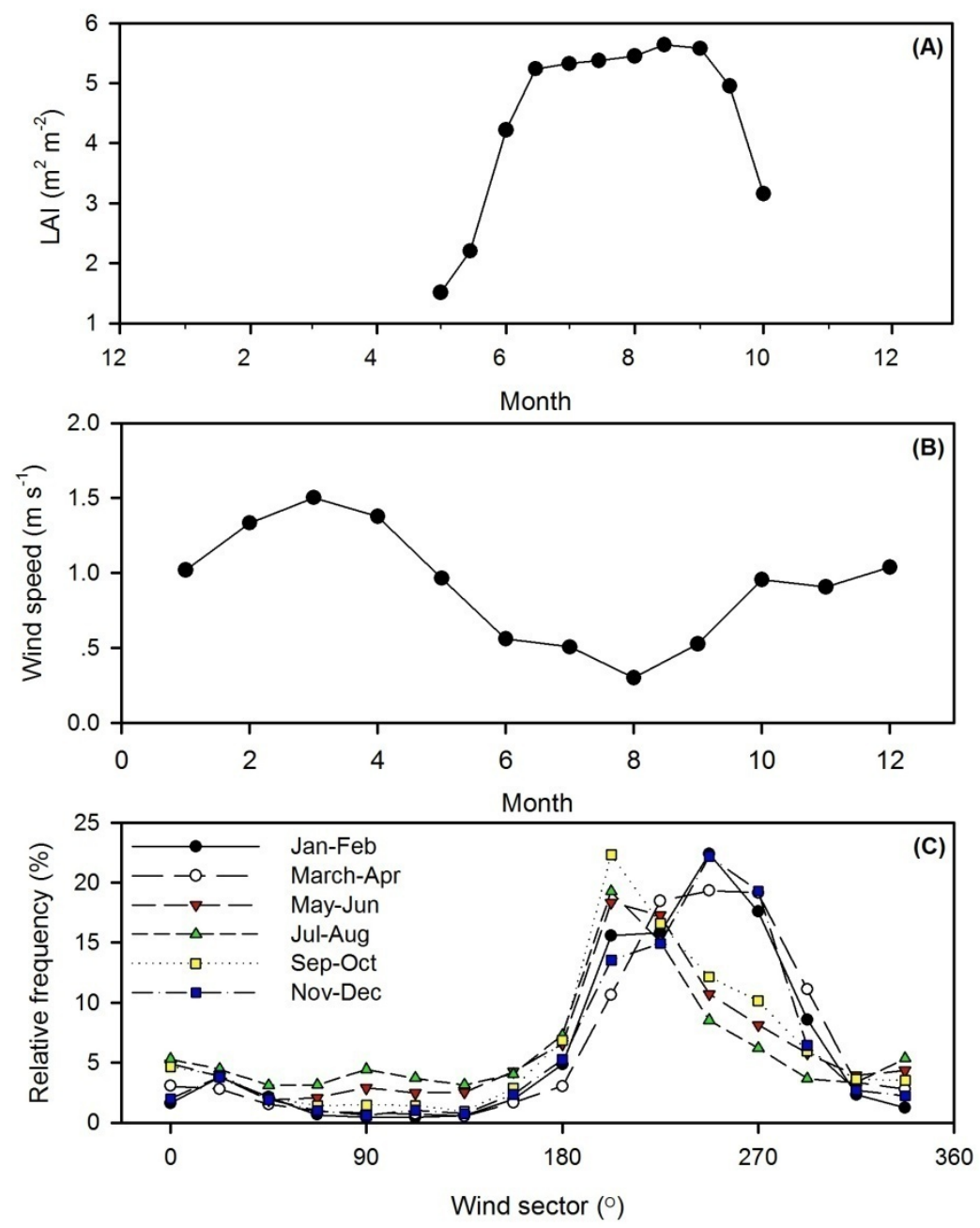
130 Table S1. Statistical summary of litterfall $\mathrm{Hg}$ concentrations and litter mass in Mt. Changbai forest

\begin{tabular}{|c|c|c|c|c|c|}
\hline $\begin{array}{l}\text { Litterfall collection } \\
\text { site }\end{array}$ & Time & Species & $\begin{array}{c}\text { Concentration } \\
\left(\mathrm{ng} \mathrm{g}^{-1}\right)\end{array}$ & $\begin{array}{l}\text { Litter mass } \\
\qquad\left(\mathrm{g} \mathrm{m}^{-2}\right)\end{array}$ & $\begin{array}{c}\text { Mass-weight } \\
\text { concentration } \\
\quad\left(\mathrm{ng} \mathrm{g}^{-1}\right)\end{array}$ \\
\hline \multirow{6}{*}{ Collector-1 } & \multirow{6}{*}{ 2013-09 } & Pinus koraiensis & 44.5 & 51.70 & \multirow{6}{*}{74.8} \\
\hline & & Acer pseudo-sieboldianum & 138.5 & 24.95 & \\
\hline & & Quercus mongolica & 170.4 & 35.60 & \\
\hline & & Fraxinus mandshurica & 44.5 & 51.70 & \\
\hline & & Tilia amurensis & 138.5 & 24.95 & \\
\hline & & Others & 170.4 & 35.60 & \\
\hline Collector-1 & 2013-10 & Acer mono & 60.2 & 1.8 & 60.2 \\
\hline \multirow{7}{*}{ Collector-2 } & \multirow{7}{*}{ 2013-09 } & Pinus koraiensis & 40.4 & 6.48 & \multirow{7}{*}{39.7} \\
\hline & & Acer pseudo-sieboldianum & 46.8 & 5.38 & \\
\hline & & Quercus mongolica & 31.2 & 9.49 & \\
\hline & & Acer mono & 58.0 & 4.81 & \\
\hline & & Fraxinus mandshurica & 34.5 & 18.54 & \\
\hline & & Tilia amurensis & 45.7 & 52.12 & \\
\hline & & Others & 36.80 & 91.16 & \\
\hline \multirow{4}{*}{ Collector-2 } & \multirow{4}{*}{ 2013-10 } & Pinus koraiensis & 34.1 & 4.4 & \multirow{4}{*}{53.3} \\
\hline & & Quercus mongolica & 30.3 & 8.8 & \\
\hline & & Acer mono & 70.7 & 5.8 & \\
\hline & & Others & 81.3 & 6.6 & \\
\hline \multirow{8}{*}{ Collector-3 } & \multirow{8}{*}{ 2013-09 } & Acer ginnala Maxim & 38.1 & 5.8 & \multirow{8}{*}{31.1} \\
\hline & & Pinus koraiensis & 29.4 & 19.0 & \\
\hline & & Acer pseudo-sieboldianum & 49.6 & 4.6 & \\
\hline & & Quercus mongolica & 32.5 & 10.8 & \\
\hline & & Acer mono & 46.9 & 6.0 & \\
\hline & & Fraxinus mandshurica & 23.4 & 1.5 & \\
\hline & & Tilia amurensis & 40.4 & 19.6 & \\
\hline & & Others & 29.8 & 283.3 & \\
\hline \multirow{2}{*}{ Collector-3 } & \multirow{2}{*}{ 2013-10 } & Pinus koraiensis & 21.7 & 1.5 & \multirow{2}{*}{35.8} \\
\hline & & Quercus mongolica & 42.3 & 3.2 & \\
\hline \multirow{6}{*}{ Collector-4 } & \multirow{6}{*}{ 2013-09 } & Pinus koraiensis & 31.4 & 70.6 & \multirow{6}{*}{34.2} \\
\hline & & Acer pseudo-sieboldianum & 47.4 & 2.7 & \\
\hline & & Quercus mongolica & 26.3 & 4.1 & \\
\hline & & Acer mono & 50.9 & 15.5 & \\
\hline & & Tilia amurensis & 43.0 & 11.8 & \\
\hline & & Others & 32.8 & 121.1 & \\
\hline \multirow{3}{*}{ Collector-4 } & \multirow{3}{*}{$2013-10$} & Pinus koraiensis & 40.1 & 19.4 & \multirow{3}{*}{64.4} \\
\hline & & Quercus mongolica & 46.0 & 21.7 & \\
\hline & & Others & 100.9 & 23.9 & \\
\hline
\end{tabular}

131 
132 Table S2. Concentration of atmospheric speciated Hg (GEM, PBM, and GOM) and isotopic

133 composition of atmospheric GEM at Mt. Changbai forest

\begin{tabular}{|c|c|c|c|c|c|c|c|c|c|c|c|c|}
\hline Sample & Sampling period & $\begin{array}{c}\text { GEM } \\
\text { conc. }\end{array}$ & $\begin{array}{l}\text { PBM } \\
\text { conc. }\end{array}$ & $\begin{array}{l}\text { GOM } \\
\text { conc. }\end{array}$ & $\delta^{202} \mathrm{Hg}$ & $\delta^{202} \mathrm{Hg}$ & $\Delta^{199} \mathrm{Hg}$ & $\Delta \Delta^{199} \mathrm{Hg}$ & $\Delta^{200} \mathrm{Hg}$ & $\Delta^{200} \mathrm{Hg}$ & $\Delta^{201} \mathrm{Hg}$ & $\Delta^{201} \mathrm{Hg}$ \\
\hline & & $\operatorname{ng~m}^{-3}$ & $\mathrm{pg} \mathrm{m}^{-3}$ & $\operatorname{pg~m^{-3}}$ & $\%$ & $2 \sigma, \%$ & $\%$ & $2 \sigma, \%$ & $\%$ & $2 \sigma, \%$ & $\%$ & $2 \sigma, \%$ \\
\hline GEM-1 & $2013 / 7 / 8$ 13:40-2013/7/9 15:30 & 1.60 & 2 & 4 & -0.06 & 0.09 & -0.05 & 0.04 & 0.01 & 0.04 & -0.01 & 0.06 \\
\hline GEM-2 & $2013 / 7 / 9$ 15:30-2013/7/10 15:30 & 1.46 & 2 & 2 & 0.35 & 0.09 & -0.05 & 0.04 & -0.04 & 0.04 & -0.12 & 0.06 \\
\hline GEM-3 & 2013/7/10 15:30-2013/7/11 15:45 & 1.23 & 4 & 2 & 0.61 & 0.09 & -0.09 & 0.04 & -0.05 & 0.04 & -0.08 & 0.06 \\
\hline GEM-4 & $2013 / 7 / 11$ 15:45-2013/7/12 15:50 & 1.14 & 5 & 2 & 0.80 & 0.09 & -0.08 & 0.04 & -0.03 & 0.04 & -0.06 & 0.06 \\
\hline GEM-5 & $2013 / 7 / 12$ 15:50-2013/7/13 16:00 & 0.91 & 6 & 1 & 0.91 & 0.09 & -0.06 & 0.04 & -0.04 & 0.04 & -0.07 & 0.06 \\
\hline GEM-6 & $2013 / 7 / 13$ 16:00-2013/7/14 15:40 & 1.32 & 4 & 2 & 0.58 & 0.09 & -0.06 & 0.04 & -0.06 & 0.04 & -0.05 & 0.06 \\
\hline GEM-7 & 2013/7/14 15:40-2013/7/15 17:10 & 1.37 & 2 & 2 & 0.58 & 0.09 & -0.08 & 0.04 & -0.05 & 0.04 & -0.04 & 0.06 \\
\hline GEM-8 & $2013 / 7 / 15$ 17:15-2013/7/16 17:30 & 1.24 & 2 & 2 & 0.08 & 0.09 & -0.04 & 0.04 & -0.04 & 0.04 & -0.04 & 0.06 \\
\hline GEM-9 & 2013/7/16 17:30-2013/7/17 18:10 & 1.57 & 5 & 1 & -0.34 & 0.09 & -0.11 & 0.04 & -0.04 & 0.04 & -0.06 & 0.06 \\
\hline GEM-10 & 2013/7/17 18:10-2013/7/18 18:35 & 1.30 & 8 & 1 & -0.01 & 0.09 & -0.08 & 0.04 & -0.05 & 0.04 & -0.04 & 0.06 \\
\hline
\end{tabular}

134

135 

litterfall $\mathrm{Hg}$ deposition of $\mathrm{Hg}$ over the world.

\begin{tabular}{|c|c|c|c|c|c|c|}
\hline \multirow[t]{2}{*}{ Region } & \multicolumn{3}{|c|}{$\begin{array}{l}\text { Litterfall } \mathrm{Hg} \text { deposition flux } \\
\qquad\left(\mu \mathrm{g} \mathrm{m}^{-2} \mathrm{yr}^{-1}\right)\end{array}$} & \multirow{2}{*}{$\begin{array}{l}\text { Forest area } \\
\qquad\left(\mathrm{km}^{2}\right)\end{array}$} & \multirow{2}{*}{$\begin{array}{l}\text { Estimated litterfall } \\
\text { deposition } \\
\left(\mathrm{Mg} \mathrm{yr}^{-1}\right)\end{array}$} & \multirow[t]{2}{*}{ Reference } \\
\hline & Range & Median & $\mathrm{N}$ & & & \\
\hline Asia & $20.9-220$ & $37.5 \pm 76.1$ & 6 & $5,775,220$ & 217 & $\begin{array}{l}\text { (Wang et al., 2009;Fu et al., 2010a;Fu } \\
\text { et al., 2010b;Zhou et al., 2013) }\end{array}$ \\
\hline North America & $3.8-30.9$ & $13.9 \pm 5.6$ & 47 & $6,847,010$ & 95 & $\begin{array}{l}\text { (Lindberg, 1996; Rea et al., } \\
\text { 1996; Grigal et al., 2000; St Louis et al., } \\
\text { 2001;Sheehan et al., 2006;Demers et } \\
\text { al., 2007;Bushey et al., 2008; Fisher } \\
\text { and Wolfe, 2012;Juillerat et al., } \\
\text { 2012; Risch et al., 2012;Benoit et al., } \\
\text { 2013) }\end{array}$ \\
\hline Europe (including Russia) & $2.7-25.2$ & $14.2 \pm 8.9$ & 6 & $10,156,300$ & 144 & $\begin{array}{c}\text { (Iverfeldt, 1991;Munthe et al., } \\
\text { 1995;Lee et al., 2000;Schwesig and } \\
\text { Matzner, 2000;Lindberg et al., } \\
\text { 2007;Larssen et al., 2008) }\end{array}$ \\
\hline South America & $43.0-184$ & $60.0 \pm 49.0$ & 9 & $9,436,410$ & 566 & $\begin{array}{c}\text { (Roulet et al., 1998;Fostier et al., } \\
\text { 2003;Mélières et al., 2003;Silva-Filho } \\
\text { et al., 2006;Teixeira et al., 2012) }\end{array}$ \\
\hline Africa & & & & $6,164,310$ & $159^{*}$ & Lack of observational data \\
\hline Oceania & & & & $1,951,370$ & $50 *$ & Lack of observational data \\
\hline Global total & & & & & 1232 & \\
\hline
\end{tabular}

(Estimated litterfall deposition: * indicates the values were calculated using the global median litterfall Hg deposition flux and the forest 
142 Table S4. A statistical summary of reported throughfall Hg deposition fluxes and estimated annual 143 throughfall $\mathrm{Hg}$ deposition of $\mathrm{Hg}$ over the world

\begin{tabular}{|c|c|c|c|c|c|c|}
\hline \multirow[t]{2}{*}{ Region } & $\begin{array}{r}\text { Throughfal } \\
\qquad(\mu\end{array}$ & $\left.\mathrm{m}^{-2} \mathrm{yr}^{-1}\right)$ & flux & \multirow{2}{*}{$\begin{array}{l}\text { Forest area } \\
\qquad\left(\mathrm{km}^{2}\right)\end{array}$} & \multirow{2}{*}{$\begin{array}{l}\text { Estimated throughfall } \\
\text { deposition }\left(\mathrm{mg} \mathrm{yr}^{-1}\right)\end{array}$} & \multirow[t]{2}{*}{ Reference } \\
\hline & Range & Median & $\mathrm{N}$ & & & \\
\hline Asia & $10.5-71.3$ & $36.8 \pm 29.9$ & 4 & $5,775,220$ & 213 & $\begin{array}{l}\text { (Wan et al., 2009; Wang et al., 2009; Fu } \\
\text { et al., 2010a;Fu et al., 2010b) }\end{array}$ \\
\hline North America & $3.8-30.9$ & $11.8 \pm 3.3$ & 10 & $6,847,010$ & 81 & $\begin{array}{l}\text { (Lindberg, 1996; Rea et al., 1996;Grigal } \\
\text { et al., 2000;St Louis et al., } \\
\text { 2001; Sheehan et al., 2006; Choi et al., } \\
\text { 2008;Fisher and Wolfe, 2012) }\end{array}$ \\
\hline $\begin{array}{l}\text { Europe (including } \\
\text { Russia) }\end{array}$ & $6.8-39.0$ & $15.2 \pm 10.7$ & 8 & $10,156,300$ & 154 & $\begin{array}{l}\text { (Iverfeldt, 1991;Munthe et al., 1995;Lee } \\
\text { et al., 2000;Schwesig and Matzner, } \\
\text { 2000;Lindberg et al., 2007; Larssen et } \\
\text { al., 2008) }\end{array}$ \\
\hline South America & 72 & 72 & 1 & $9,436,410$ & 679 & (Fostier et al., 2000) \\
\hline Africa & & & & $6,164,310$ & $160^{*}$ & Lack of observational data \\
\hline Oceania & & & & $1,951,370$ & $51^{*}$ & Lack of observational data \\
\hline Global total & & & & & 1338 & \\
\hline $\begin{array}{l}\text { (Estimated throughfal } \\
\text { forest area in these res }\end{array}$ & $\begin{array}{l}\text { deposition: } \\
\text { ions) }\end{array}$ & indicates th & values & were calcula & using the global media & throughfall $\mathrm{Hg}$ deposition flux and the \\
\hline
\end{tabular}


149 Table S5. A statistical summary of reported forest soil emission fluxes and estimated annual forest soil emission fluxes over the world

151

\begin{tabular}{|c|c|c|c|c|c|c|}
\hline \multirow[t]{2}{*}{ Region } & $\begin{array}{l}\text { Forest soil } \\
\qquad \mu\end{array}$ & $\begin{array}{l}\text { Ig emission } \\
\left.\mathrm{m}^{-2} \mathrm{yr}^{-1}\right)\end{array}$ & lux & Forest area & Estimated forest soil & \multirow[t]{2}{*}{ Reference } \\
\hline & Range & Median & $\mathrm{N}$ & & & \\
\hline Asia & $3.3-81.2$ & $30.4 \pm 23.5$ & 11 & $5,775,220$ & 176 & $\begin{array}{c}\text { (Wang et al., 2006; Fu et al., 2008; Fu et al., } \\
\text { 2012;Ma et al., 2013) }\end{array}$ \\
\hline
\end{tabular}

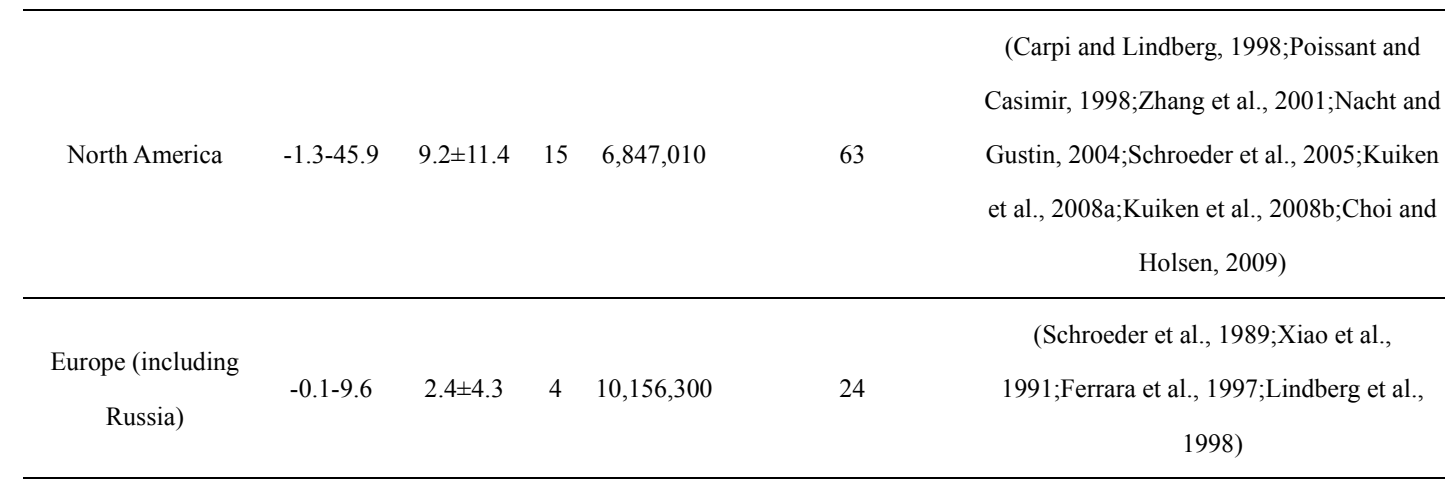

\begin{tabular}{|c|c|c|c|c|c|c|}
\hline South America & 6.0 & 6.0 & 1 & $9,436,410$ & 57 & (Carpi et al., 2014) \\
\hline Africa & & & & $6,164,310$ & $47 *$ & Lack of observational data \\
\hline Oceania & & & & $1,951,370$ & $15^{*}$ & Lack of observational data \\
\hline
\end{tabular}

Global total

381

152 (Estimated forest soil emission: * indicates the values were calculated using the global median forest soil Hg emission flux and the forest area in these regions) 


\section{Reference:}

Benoit, J. M., Cato, D. A., Denison, K. C., and Moreira, A. E.: Seasonal Mercury Dynamics in a New England Vernal Pool, Wetlands, 33, 887-894, DOI 10.1007/s13157-013-0447-4, 2013.

Bushey, J. T., Nallana, A. G., Montesdeoca, M. R., and Driscoll, C. T.: Mercury dynamics of a northern hardwood canopy, Atmos Environ, 42, 6905-6914, DOI 10.1016/j.atmosenv.2008.05.043, 2008.

Carpi, A., and Lindberg, S. E.: Application of a Teflon (TM) dynamic flux chamber for quantifying soil mercury flux: Tests and results over background soil, Atmos Environ, 32, 873-882, Doi 10.1016/S1352-2310(97)00133-7, 1998.

Carpi, A., Fostier, A. H., Orta, O. R., dos Santos, J. C., and Gittings, M.: Gaseous mercury emissions from soil following forest loss and land use changes: Field experiments in the United States and Brazil, Atmos Environ, 96, 423-429, DOI 10.1016/j.atmosenv.2014.08.004, 2014.

Choi, H. D., Sharac, T. J., and Holsen, T. M.: Mercury deposition in the Adirondacks: A comparison between precipitation and throughfall, Atmos Environ, 42, 1818-1827, DOI 10.1016/j.atmosenv.2007.11.036, 2008.

Choi, H. D., and Holsen, T. M.: Gaseous mercury fluxes from the forest floor of the Adirondacks, Environ Pollut, 157, 592-600, DOI 10.1016/j.envpol.2008.08.020, 2009.

Demers, J. D., Driscoll, C. T., Fahey, T. J., and Yavitt, J. B.: Mercury cycling in litter and soil in different forest types in the Adirondack region, New York, USA, Ecol Appl, 17, 1341-1351, Doi 10.1890/06-1697.1, 2007.

Ferrara, R., Maserti, B. E., Andersson, M., Edner, H., Ragnarson, P., and Svanberg, S.: Mercury degassing rate from mineralized areas in the Mediterranean basin, Water Air Soil Poll, 93, 59-66, Doi 10.1023/A:1022107205659, 1997.

Fisher, L. S., and Wolfe, M. H.: Examination of mercury inputs by throughfall and litterfall in the Great Smoky Mountains National Park, Atmos Environ, 47, 554-559, DOI 10.1016/j.atmosenv.2011.10.017, 2012.

Foken, T.: Micrometeorology, Springer-Verlag, Berlin, Heidelberg, 2008.

Fostier, A. H., Forti, M. C., Guimaraes, J. R. D., Melfi, A. J., Boulet, R., Santo, C. M. E., and Krug, F. J.: Mercury fluxes in a natural forested Amazonian catchment (Serra do Navio, Amapa State, Brazil), Sci Total Environ, 260, 201-211, Doi 10.1016/S0048-9697(00)00564-7, 2000.

Fostier, A. H., Cecon, K., and Forti, M. C.: Urban influence on litterfall trace metals fluxes in the Atlantic forest of Sao Paulo (Brazil), J Phys Iv, 107, 491-494, Doi 10.1051/Jp4:20030348, 2003.

Fu, X., Feng, X., Zhu, W., Rothenberg, S., Yao, H., and Zhang, H.: Elevated atmospheric deposition and dynamics of mercury in a remote upland forest of southwestern China, Environ Pollut, 158, 2324-2333, 10.1016/j.envpol.2010.01.032, 2010a.

Fu, X. W., Feng, X. B., and Wang, S. F.: Exchange fluxes of $\mathrm{Hg}$ between surfaces and atmosphere in the eastern flank of Mount Gongga, Sichuan province, southwestern China, J Geophys Res-Atmos, 113, Artn D20306 Doi 10.1029/2008jd009814, 2008.

Fu, X. W., Feng, X., Dong, Z. Q., Yin, R. S., Wang, J. X., Yang, Z. R., and Zhang, H.: Atmospheric gaseous elemental mercury (GEM) concentrations and mercury depositions at a high-altitude mountain peak in south China, Atmos Chem Phys, 10, 2425-2437, 2010b.

Fu, X. W., Feng, X. B., Zhang, H., Yu, B., and Chen, L. G.: Mercury emissions from natural surfaces highly impacted by human activities in Guangzhou province, South China, Atmos Environ, 54, 185-193, DOI 10.1016/j.atmosenv.2012.02.008, 2012. 
Garratt, J. R.: The atmospheric boundary layer, Cambridge University Press, Cambridge, UK, 1992.

Grigal, D. F., Kolka, R. K., Fleck, J. A., and Nater, E. A.: Mercury budget of an upland-peatland watershed, Biogeochemistry, 50, 95-109, Doi 10.1023/A:1006322705566, 2000.

Huang, H. Y., Hall, A., and Teixeira, J.: Evaluation of the WRF PBL Parameterizations for Marine Boundary Layer Clouds: Cumulus and Stratocumulus, Mon Weather Rev, 141, 2265-2271, 10.1175/Mwr-D-12-00292.1, 2013.

Iverfeldt, A.: Mercury in forest canopy throughfall water and its relation to atmospheric deposition, Water Air Soil Poll, 56, 553-564, Doi 10.1007/Bf00342299, 1991.

Juillerat, J. I., Ross, D. S., and Bank, M. S.: Mercury in litterfall and upper soil horizons in forested ecosystems in Vermont, USA, Environ Toxicol Chem, 31, 1720-1729, Doi 10.1002/Etc.1896, 2012. Kaimal, J. C., and Finnigan, J. J.: Atmospheric Boundary Layer Flows, Oxford University Press, New York, Oxford, 1994. Research and Forecasting (WRF) model under the aspect of offshore wind energy applicationspart II: boundary layer height and atmospheric stability, Wind Energy, 18, 1291-1302, 10.1002/we.1765, 2015. Kuiken, T., Gustin, M., Zhang, H., Lindberg, S., and Sedinger, B.: Mercury emission from terrestrial background surfaces in the eastern USA. II: Air/surface exchange of mercury within forests from South Carolina to New England, Appl Geochem, 23, 356-368, DOI 10.1016/j.apgeochem.2007.12.007, 2008a.

Kuiken, T., Zhang, H., Gustin, M., and Lindberg, S.: Mercury emission from terrestrial background surfaces in the eastern USA. Part I: Air/surface exchange of mercury within a southeastern deciduous forest (Tennessee) over one year, Appl Geochem, 23, 345-355, DOI 10.1016/j.apgeocliem.2007.12.006, 2008 b. catchment in southeast Norway, Sci Total Environ, 404, 290-296, DOI 10.1016/j.scitotenv.2008.03.013, 2008.

Lee, Y. H., Bishop, K. H., and Munthe, J.: Do concepts about catchment cycling of methylmercury and mercury in boreal catchments stand the test of time? Six years of atmospheric inputs and runoff export at Svartberget, northern Sweden, Sci Total Environ, 260, 11-20, Doi 10.1016/S0048-9697(00)00538-6, 2000 . mercury in deposition, Ambio, 36, 19-32, 2007.

Lindberg, S. E.: Forests and the global biogeochemical cycle of mercury: The importance of understanding air/vegetation exchange processes, Nato Asi 2, 21, 359-380, 1996.

Lindberg, S. E., Hanson, P. J., Meyers, T. P., and Kim, K. H.: Air/surface exchange of mercury vapor over forests - The need for a reassessment of continental biogenic emissions, Atmos Environ, 32, 895-908, Doi 10.1016/S1352-2310(97)00173-8, 1998.

Mélières, M. A., Pourchet, M., Charles-Dominique, P., and Gaucher, P.: Mercury in canopy leaves of French Guiana in remote areas, Sci Total Environ, 311, 261-267, Doi 10.1016/S0048-9697(03)00142-6, 2412003.

242 Ma, M., Wang, D. Y., Sun, R. G., Shen, Y. Y., and Huang, L. X.: Gaseous mercury emissions from 243 subtropical forested and open field soils in a national nature reserve, southwest China, Atmos Environ, 64, 116-123, DOI 10.1016/j.atmosenv.2012.09.038, 2013. 
Munthe, J., Hultberg, H., and Iverfeldt, A.: Mechanisms of Deposition of Methylmercury and Mercury to Coniferous Forests, Water Air Soil Poll, 80, 363-371, Doi 10.1007/Bf01189686, 1995.

Nacht, D. M., and Gustin, M. S.: Mercury emissions from background and altered geologic units throughout Nevada, Water Air Soil Poll, 151, 179-193, Doi 10.1023/B:Wate.0000009907.49577.A8, 2004.

Poissant, L., and Casimir, A.: Water-air and soil-air exchange rate of total gaseous mercury measured at background sites, Atmos Environ, 32, 883-893, Doi 10.1016/S1352-2310(97)00132-5, 1998.

Raupach, M. R.: Simplified Expressions for Vegetation Roughness Length and Zero-Plane Displacement as Functions of Canopy Height and Area Index, Bound-Lay Meteorol, 71, 211-216, Doi 10.1007/Bf00709229, 1994.

Rea, A. W., Keeler, G. J., and Scherbatskoy, T.: The deposition of mercury in throughfall and litterfall in the lake champlain watershed: A short-term study, Atmos Environ, 30, 3257-3263, Doi 10.1016/1352-2310(96)00087-8, 1996.

Risch, M. R., DeWild, J. F., Krabbenhoft, D. P., Kolka, R. K., and Zhang, L. M.: Litterfall mercury dry deposition in the eastern USA, Environ Pollut, 161, 284-290, DOI 10.1016/j.envpol.2011.06.005, 2012. Roulet, M., Lucotte, M., Saint-Aubin, A., Tran, S., Rheault, I., Farella, N., Da Silva, E. D., Dezencourt, J., Passos, C. J. S., Soares, G. S., Guimaraes, J. R. D., Mergler, D., and Amorim, M.: The geochemistry of mercury in central Amazonian soils developed on the Alter-do-Chao formation of the lower Tapajos River Valley, Para state, Brazil, Sci Total Environ, 223, 1-24, Doi 10.1016/S0048-9697(98)00265-4, 1998.

Schroeder, W. H., Munthe, J., and Lindqvist, O.: Cycling of Mercury between Water, Air, and Soil Compartments of the Environment, Water Air Soil Poll, 48, 337-347, 1989.

Schroeder, W. H., Beauchamp, S., Edwards, G., Poissant, L., Rasmussen, P., Tordon, R., Dias, G., Kemp, J., Van Heyst, B., and Banic, C. M.: Gaseous mercury emissions from natural sources in Canadian landscapes, J Geophys Res-Atmos, 110, Artn D18302

Doi 10.1029/2004jd005699, 2005.

Schwesig, D., and Matzner, E.: Pools and fluxes of mercury and methylmercury in two forested catchments in Germany, Sci Total Environ, 260, 213-223, Doi 10.1016/S0048-9697(00)00565-9, 2000.

Sheehan, K. D., Fernandez, I. J., Kahl, J. S., and Amirbahman, A.: Litterfall mercury in two forested watersheds at Acadia National Park, Maine, USA, Water Air Soil Poll, 170, 249-265, DOI 10.1007/s11270-006-3034-y, 2006.

Shi, T. T., Guan, D. X., Wang, A. Z., Wu, J. B., Jin, C. J., and Han, S. J.: Comparison of three models to estimate evapotranspiration for a temperate mixed forest, Hydrol Process, 22, 3431-3443, 10.1002/hyp.6922, 2008.

Silva-Filho, E. V., Machado, W., Oliveira, R. R., Sella, S. M., and Lacerda, L. D.: Mercury deposition through litterfall in an Atlantic Forest at Ilha Grande, southeast Brazil, Chemosphere, 65, 2477-2484, DOI 10.1016/j.chemosphere.2006.04.053, 2006.

Simpson, I. J., Thurtell, G. W., Neumann, H. H., Den Hartog, G., and Edwards, G. C.: The validity of similarity theory in the roughness sublayer above forests, Bound-Lay Meteorol, 87, 69-99, Doi 10.1023/A:1000809902980, 1998.

St Louis, V. L., Rudd, J. W. M., Kelly, C. A., Hall, B. D., Rolfhus, K. R., Scott, K. J., Lindberg, S. E., and Dong, W.: Importance of the forest canopy to fluxes of methyl mercury and total mercury to boreal ecosystems, Environmental Science \& Technology, 35, 3089-3098, Doi 10.1021/Es001924p, 2001.

Teixeira, D. C., Montezuma, R. C., Oliveira, R. R., and Silva, E. V.: Litterfall mercury deposition in 
Atlantic forest ecosystem from SE - Brazil, Environ Pollut, 164, 11-15, DOI 10.1016/j.envpol.2011.10.032, 2012.

291 Wan, Q., Feng, X. B., Lu, J., Zheng, W., Song, X. J., Li, P., Han, S. J., and Xu, H.: Atmospheric mercury in Changbai Mountain area, northeastern China II. The distribution of reactive gaseous mercury and particulate mercury and mercury deposition fluxes, Environ Res, 109, 721-727, DOI 10.1016/j.envres.2009.05.006, 2009. Wang, D. Y., He, L., Shi, X. J., Wei, S. Q., and Feng, X. B.: Release flux of mercury from different environmental surfaces in Chongqing, China, Chemosphere, 64, 1845-1854, DOI 10.1016/j.chemosphere.2006.01.054, 2006. subtropical forested catchments, southwest China, Environ Pollut, 157, 801-808, DOI 10.1016/j.envpol.2008.11.018, 2009.

Xiao, Z. F., Munthe, J., Schroeder, W. H., and Lindqvist, O.: Vertical Fluxes of Volatile Mercury over Forest Soil and Lake Surfaces in Sweden, Tellus B, 43, 267-279, DOI 10.1034/j.1600-0889.1990.t01-1-00009.x-11, 1991.

304 Zhang, H., Lindberg, S. E., Marsik, F. J., and Keeler, G. J.: Mercury air/surface exchange kinetics of background soils of the Tahquamenon River watershed in the Michigan Upper Peninsula, Water Air Soil Poll, 126, 151-169, Doi 10.1023/A:1005227802306, 2001.

307 Zhou, J., Feng, X. B., Liu, H. Y., Zhang, H., Fu, X. W., Bao, Z. D., Wang, X., and Zhang, Y. P.: 308 Examination of total mercury inputs by precipitation and litterfall in a remote upland forest of 309 Southwestern China, Atmos Environ, 81, 364-372, DOI 10.1016/j.atmosenv.2013.09.010, 2013. 